Alda Patrícia Marques Portugal ${ }^{1}$

Isabel Maria Marques Alberto ${ }^{1}$

\title{
Caracterização da comunicação entre progenitores e filhos adolescentes: estudo das variáveis sociodemográficas
}

\author{
Characterization of parent-adolescent communication: \\ a study of socio-demographic variables
}

${ }^{1}$ Faculdade de Psicologia e de Ciências da Educação, Universidade de Coimbra. R. Colégio Novo 6153.3001802 Coimbra Portugal. aaldaportugal@gmail.com

\begin{abstract}
Parent-adolescent communication seems to depend largely of some socio-demographic characteristics. The main goal of this research is the analysis of parent-adolescent perception about their communication considering some socio-demographic variables: sex, residence, socioeconomic status, education and family composition. A quantitative cross-sectional design was used to analyze the perception of 336 parents and 268 children about the quality of communication using the Perception Scale of Parenting Communication.: It was revealed that: boys tend to share their problems mostly with fathers; mothers have better perception of communication; there weren't statistical differences on communicational conflict in the sex variable; members of high socioeconomic classes and urban contexts have better perception about communication; and adolescents of post-divorce families tend to share their problems mostly with mothers than adolescents of intact nuclear families. Considering the influence of some socio-demographic variables on parent-adolescent communication, it is possible design concrete clinical interventions. It suggests the need to pay attention to particular contexts (rural places and low socioeconomic classes). These results demystify the belief that post-divorce families have more difficulties than nuclear intact families.
\end{abstract}

Key words Communication, Parents, Adolescents
Resumo A comunicação estabelecida entre progenitores e filhos adolescentes parece depender, em grande medida, de algumas características sociodemográficas. Objetivo: Análise da comunicação em famílias com filhos adolescentes, considerando as variáveis: sexo, área de residência, nível socioeconómico, escolaridade e estrutura familiar. Método: Através de um desenho quantitativo/ transversal, aplicou-se a Escala de Avaliação da Comunicação na Parentalidade a 336 pais e 268 adolescentes. Resultados: Verifica-se que: os rapazes tendem a partilhar os seus problemas com os progenitores do sexo masculino; as mães destacam-se na perceção de maior expressão do afeto/ apoio emocional; não se registram diferenças significativas ao nível do sexo no conflito comunicacional; membros de classes socioeconómicas altas e de contextos urbanos percepcionam melhor comunicação; e filhos de famílias pós-divórcio revelam partilhar mais os seus problemas com as mães do que filhos de agregados monoparentais. Conclusões: A influência de algumas variáveis sociodemográficas sobre a comunicação permite o desenho de intervenções clínicas concretas, sugerindo a necessidade de se prestar maior atenção a contextos particulares (classes rurais e socioeconómicas baixas). Estes resultados desmistificam a crença de que as famílias pós-divórcio têm mais dificuldades do que famílias nucleares intactas.

Palavras-chave Comunicação, Relações parentofiliais, Adolescentes 


\section{Introdução}

A adolescência é uma fase decisiva na construção da identidade pessoal e das relações que o indivíduo mantém com os seus pares, com os adultos e com o meio que o rodeia ${ }^{1}$. A comunicação desempenha um papel central na gestão dos desafios e concretização das tarefas inerentes a esta etapa do ciclo vital ${ }^{2}$, tornando essencial a análise dos padrões comunicacionais, entre progenitores e filhos, que caraterizam esta fase de desenvolvimento individual e familiar.

Apesar de a vivência da adolescência variar em função dos contextos sociais e dos momentos históricos ${ }^{1}$, é possível identificar características incontornáveis desta fase da vida, nomeadamente, a necessidade de promover transformações de cariz estrutural na família, a renegociação de padrões de relacionamento e de comunicação e a aceitação de transformações ao nível da personalidade, tanto dos progenitores como do(s) filho $(s)^{3}$. Estas transformações, equivalentes a uma metamorfose familiar, podem acarretar dificuldades para os elementos da família, motivo pelo qual a adolescência é considerada um período de crise que ativa a mudança ${ }^{3}$.

As principais características desta etapa de desenvolvimento são: (a) o despertar para a sexualidade e para as transformações corporais por parte do adolescente, (b) a tomada de consciência e o desenvolvimento de um pensamento abstrato sobre caraterísticas pessoais que formam a identidade individual (crise identitária), (c) o processo de autonomização e diferenciação em relação ao núcleo familiar, e (d) a abordagem de temas relacionados com a vinculação, separação e perda ${ }^{3,4}$. A resolução inadequada destas tarefas pode ter implicações no desenvolvimento individual e familiar ${ }^{2}$.

A análise da comunicação familiar é fundamental na compreensão do processo de adaptação de progenitores e de filhos à adolescência ${ }^{5}$. A comunicação, enquanto processo contínuo de transmissão de informação entre progenitores e filhos permite atender e dar resposta às exigências específicas desta etapa do ciclo vital ${ }^{2,3}$. Tomé et al. ${ }^{6}$ defendem que o processo de comunicação parento-filial influencia positivamente a saúde dos adolescentes: quando a comunicação com os progenitores é percepcionada pelos adolescentes como sendo agradável, estes tendem a manifestar maior satisfação com a vida e menos sintomas negativos em termos de saúde.

De acordo com a literatura ${ }^{2,7}$, a percepção de conflito constante entre progenitores e filhos, nesta etapa do ciclo vital, é um mito, não correspondendo aos discursos e às representações sociais. Segrin e Flora ${ }^{5}$ referem que as interações parento-filiais conflituosas que se verificam nesta fase desenvolvimental são normativas e, inclusivamente, promotoras de reajustes ao nível das expectativas e da renegociação de papéis. Laursen e Collins ${ }^{8}$ consideram, inclusivamente, que o conflito na adolescência tende a reforçar os laços afetivos entre progenitores e filhos a curto e a longo prazo, estimulando a comunicação sobre a relação.

As características específicas desta fase de desenvolvimento poderão explicar a quantidade de pesquisas existentes que se debruçam sobre o estudo da adolescência ${ }^{8-10}$. Os estudos sobre a comunicação familiar, enquadrada no exercício da parentalidade, sugerem a existência de diferenças de género relativamente ao estabelecimento da comunicação, existindo indicadores de que os adolescentes de ambos os sexos, tendem a procurar mais as mães do que os pais para comunicar $^{8-10}$. Jiménez e Delgado ${ }^{10}$ verificaram que o nível de conflituosidade entre progenitores e filhos é percecionado de forma mais intensa por parte dos rapazes do que das adolescentes.

Também as características sociodemográficas da família parecem ter influência na gestão comunicacional $^{3}$, nomeadamente: (a) o nível de instrução, isto é, quanto menor for a escolaridade dos progenitores menor tenderá a ser a qualidade das interações comunicacionais ${ }^{10}$; e (b) o tipo de estrutura familiar ${ }^{11}$. Considerando as configurações familiares, Carlsund et al. ${ }^{11}$ verificaram que os adolescentes de famílias monoparentais e de famílias pós-divórcio (em regime de guarda partilhada) tendem a apresentar mais queixas relacionadas com a saúde e a reportar menor bem-estar, comparativamente aos adolescentes de famílias nucleares intactas. Embora a literatura não seja absolutamente consensual a este respeito, importa fazer a distinção entre famílias pós-divórcio, famílias monoparentais e famílias reconstituídas: as primeiras correspondem a composições cujo núcleo familiar se desintegrou por opção dos elementos do casal (separação ou divórcio conjugal), mas, apesar disso, o exercício da parentalidade continua a ser exercido, em geral, por ambos os progenitores ${ }^{3,12}$; as famílias monoparentais caracterizam-se pelo facto do exercício da parentalidade ser desempenhado exclusivamente por um único progenitor, dado o outro se encontrar voluntária ou involuntariamente ausente (e.g., morte, divórcio) ${ }^{13}$; e, por fim, as famílias reconstituídas dizem respeito a configu- 
rações familiares cujos elementos tiveram outras famílias (num passado mais ou menos próximo) e que agora estão, em parte, reunidos num novo sistema ${ }^{13}$. Desta forma, as famílias reconstituídas resultam, necessariamente, de constelações familiares pós-divórcio.

A atual necessidade de desenvolver pesquisa sobre a comunicação entre pais e filhos adolescentes resulta das transformações que a vivência da adolescência tem sofrido nas últimas décadas, tanto a nível social (e.g., independência tardia relativamente ao agregado familiar), como a nível tecnológico (e.g., banalização do uso da internet, telemóvel, redes sociais), influenciando a forma como os progenitores exercem a parentalidade nesta fase $\mathrm{e}^{1}$. Particularmente em Portugal, a investigação sobre a comunicação estabelecida entre progenitores e filhos adolescentes é escassa, refletindo uma lacuna nos instrumentos de avaliação psicológica que avaliem especificamente os padrões comunicacionais.

A presente pesquisa tem como objetivo geral analisar as perceções de progenitores e de adolescentes sobre a comunicação estabelecida na relação parento-filial. Considerando este objetivo, foram definidos três subobjetivos: (1) identificar diferenças na comunicação parentofilial em função do sexo dos progenitores e dos adolescentes $^{9,10}$; (2) analisar se o nível de conflito com as figuras parentais é independente do sexo dos adolescentes ${ }^{10}$; e (3) analisar a influência das variáveis escolaridade, área de residência, nível socioeconómico e estrutura familiar de progenitores e adolescentes na perceção dos padrões comunicacionais entre pais e filhos ${ }^{3}$.

\section{Método}

\section{Amostra}

O cálculo amostral foi realizado com base no total da população portuguesa com idades entre zero e 14 anos $(\mathrm{N}=1.572 .329)$, e o total da população portuguesa com idades entre 15 e 64 anos $(\mathrm{N}=6.979 .785)$, contemplando um erro amostral de 5\%. Desta forma, o valor ideal da amostra obtido corresponde a 385 progenitores e a 309 adolescentes.

A amostra total foi constituída por 604 sujeitos distribuídos por duas amostras específicas: 268 adolescentes com idades entre 12 e 16 anos $(M=13.62, D P=1.31)$ e 336 progenitores com idades entre 30 e 58 anos $(M=43.91, D P=5.40)$. As amostras dos adultos e dos adolescentes são independentes, não havendo relação de parentesco entre si.

A amostra dos adolescentes é composta por $123(45.9 \%)$ adolescentes do sexo masculino e $145(54.1 \%)$ do sexo feminino. Considerando a área de residência, 20 adolescentes (7.5\%) vivem num contexto predominantemente urbano, 139 (51.9\%) num contexto medianamente urbano e $109(40.7 \%)$ num contexto predominantemente rural. Ao nível do estatuto socioeconómico, calculado com base nos indicadores disponíveis no Instituto Nacional de Estatística relativos às profissões e aos rendimentos dos progenitores, $17(6.3 \%)$ adolescentes pertencem à classe socioeconómica baixa, $230(85.8 \%)$ à classe média e 16 $(6 \%)$ à classe alta. No que respeita à escolaridade, dois $(0.7 \%)$ adolescentes frequentam o $4^{\circ}$ ano, $63(23.5 \%)$ frequentam o $6^{\circ}$ ano, $188(70.1 \%)$ estão no $9^{\circ}$ ano e 15 (5.6\%) frequentam o $12^{\circ}$ ano. Relativamente à estrutura familiar, verificase que $203(75.7 \%)$ adolescentes vivem em agregados familiares intactos, 32 (11.9\%) em famílias pós-divórcio, 28 (10.4\%) adolescentes vivem em famílias reconstituídas e, por fim, cinco (1.9\%) integram famílias monoparentais.

A amostra dos progenitores é composta por 57 pais $(17 \%)$ e 279 mães (83\%), verificando-se que $116(34.5 \%)$ progenitores vivem num contexto predominantemente urbano, 168 (50\%) num contexto medianamente urbano e 44 (13.1\%) habitam num contexto predominantemente rural. Relativamente ao nível socioeconómico, 226 sujeitos $(67.3 \%)$ enquadram-se no nível socioeconómico médio, 88 (26.2\%) pertencem ao nível socioeconómico baixo e 18 (5.4\%) estão no nível socioeconómico alto. Considerando a escolaridade, $31(9.2 \%)$ progenitores completaram o $4^{\mathrm{a}}$ ano, 25 (7.4\%) completaram o $6^{\circ}$ ano, 46 (13.7\%) concluíram o $9^{\circ}$ ano, 54 (16.1\%) terminaram o $12^{\circ}$ ano, $112(33.3 \%)$ têm uma licenciatura e 49 realizaram uma pós-graduação (mestrado: $n=$ 30, 8.9\%; doutoramento: $n=19,5.7 \%$ ). A maior parte dos progenitores integram famílias nucleares intactas $(n=281,83.6 \%), 38(11.3 \%)$ vivem em famílias pós-divórcio, 12 (3.6\%) pertencem a um núcleo familiar monoparental e cinco (1.5\%) vivem em famílias reconstituídas.

\section{Instrumentos}

\section{Ficha de dados sociodemográficos}

Trata-se de um questionário breve que inclui questões relacionadas com o sexo (masculino, feminino), idade (em anos), parentesco (pai, mãe, filho, filha), área de residência (predomi- 
nantemente urbano, medianamente urbano, predominantemente rural), estatuto socioeconómico (alto, médio, baixo) e tipo de composição familiar (nuclear, pós-divórcio, monoparental, reconstituída).

\section{Escala de Avaliação da Comunicação na Parentalidade (COMPA)}

A Escala de Avaliação da Comunicação na Parentalidade (COMPA) ${ }^{14}$ é um instrumento de autorrelato que tem como objetivo avaliar as perceções sobre os padrões de comunicação entre progenitores e filhos através de uma escala de tipo Likert com cinco níveis $(1=$ Nunca; $2=$ Raramente; $3=$ Às vezes; $4=$ Muitas vezes; $5=$ Sempre).

A versão da escala COMPA para os adolescentes entre os 12 e os 16 anos tem 39 itens distribuídos por cinco subescalas ${ }^{14}$, nomeadamente: disponibilidade parental para a comunicação, $\alpha$ .865; partilha/confiança de filhos para progenitores, $\alpha$ : .873; expressão afetiva/suporte emocional, $\alpha$ : .838; metacomunicação, $\alpha$ : .805; padrões negativos de comunicação, $\alpha$ : .650. A versão dos adolescentes é respondida de forma separada para o pai e para a mãe. Por sua vez, a versão parental da escala COMPA é composta por 44 itens distribuídos por cinco subescalas ${ }^{14}$ : expressão afetiva/suporte emocional, $\alpha$ : .821; disponibilidade parental para a comunicação, $\alpha$ : .732; metacomunicação, $\alpha:$.725; partilha/confiança de progenitores para filhos, $\alpha$ : .753; partilha/confiança de filhos para progenitores, $\alpha: .615$.

A cotação do instrumento deve ser realizada por subescala, não existindo um resultado global. Para tal, deve fazer-se o somatório dos itens por subescala e dividir os totais obtidos, em cada subescala, pelo número de itens que a compõem. Atendendo às especificidades de algumas subescalas (existência de itens formulados pela negativa), quanto mais elevada for a pontuação, melhor tende a ser a perceção sobre a comunicação estabelecida entre progenitores e filhos ${ }^{14}$

\section{Procedimento}

O presente estudo foi divulgado por diversas escolas públicas. Entre aquelas que aceitaram participar na presente investigação, distribuiu-se, pelos alunos, um documento informativo sobre o estudo e os seus objetivos, assim como, os consentimentos informados para serem assinados pelos respetivos progenitores. O protocolo de avaliação foi respondido em contexto de sala de aula. A administração do protocolo aos proge- nitores foi feita de duas formas: em formato de papel e lápis ( $n=171$, amostra de conveniência) e numa versão online ( $n=165$, criação de um website para o efeito). A opção por dois grupos amostrais (um de progenitores e um de adolescentes), sem grau de parentesco entre si, teve por base a possibilidade de maior acesso a sujeitos independentes, permitindo um aumento considerável da dimensão da amostra.

A pesquisa foi realizada de acordo com os princípios éticos básicos da Declaração de Helsinque (versão alterada na 55a Assembleia, em Tóquio, Japão, 2004), estabelecendo-se o consentimento informado e a resguarda da confidencialidade e anonimato dos respondentes. O presente trabalho foi submetido a avaliação e, posteriormente, aprovado pela Direção de Serviços de Inovação Educativa, um departamento do governo português que analisa os pressupostos éticos de pesquisas realizadas com crianças.

\section{Resultados}

Subobjetivo 1: Identificar diferenças na comunicação parento-filial em função do sexo dos progenitores e dos adolescentes

Para analisar a influência da variável sexo de progenitores e filhos na comunicação parento-filial recorreu-se ao teste de comparação de médias $t$ de Student para amostras independentes. Estes resultados poderão ser consultados na Tabela 1 .

\section{Resultados na amostra dos adolescentes}

Registrou-se apenas uma diferença estatisticamente significativa na dimensão confiançal partilha comunicacional de filhos para progenitores nas respostas dos filhos em relação aos progenitores $[t(245)=3.223, p=.001]$, com uma magnitude do efeito pequena $\left(\eta^{2}=.04\right)^{15}$. Em média, os adolescentes do sexo masculino partilham os seus problemas e confiam mais nos pais $(M=$ $3.26, D P=.72)$ do que nas mães $(M=2.95, D P$ $=.80)$. Por outro lado, e apesar de este resultado não ser estatisticamente significativo $[t(259)=$ $-1.746, p=.082]$, verificou-se que as adolescentes $(M=3.82, D P=.78)$, em comparação com os rapazes $(M=3.65, D P=.78)$, tendem a partilhar as suas dificuldades e a confiar mais nas mães do que nos pais.

Resultados na amostra dos progenitores

Obtiveram-se dois valores estatisticamente significativos, nomeadamente ao nível da subes- 


\begin{tabular}{|c|c|c|c|c|c|c|}
\hline $\begin{array}{l}\text { Sub-dimensão/ } \\
\text { Nome }\end{array}$ & & M & DP & $\mathrm{t}(\mathrm{gl})$ & $\mathrm{p}$ & $\begin{array}{c}\text { 95\% IC } \\
\text { (baixo; alto) }\end{array}$ \\
\hline \multicolumn{7}{|l|}{ COMPA-A: filhos em relação ao pai $(n=138)$} \\
\hline \multirow[t]{2}{*}{ I. Disponibilidade Parental para a Comunicação } & Masc. & 3.99 & .69 & $.234(251)$ & .815 & $-2.17764 ; 2.76486$ \\
\hline & Femin. & 3.97 & .73 & & & \\
\hline \multirow[t]{2}{*}{ II. Confiança/Partilha de Filhos para Progenitores } & Masc. & 3.26 & .72 & $3.223(242)$ & $.001^{*}$ & $.85211 ; 3.53080$ \\
\hline & Femin. & 2.95 & .80 & & & \\
\hline \multirow[t]{2}{*}{ III. Expressão do Afeto e Apoio Emocional } & Masc. & 3.81 & .89 & $.702(255)$ & .483 & $-.72345 ; 1.52457$ \\
\hline & Femin. & 3.73 & .93 & & & \\
\hline \multirow[t]{2}{*}{ IV. Metacomunicação } & Masc. & 3.64 & .68 & $.537(247)$ & .592 & $-1.10302 ; 1.93043$ \\
\hline & Femin. & 3.60 & .67 & & & \\
\hline \multirow[t]{2}{*}{ V. Padrão Comunicacional Negativo } & Masc. & 3.91 & .61 & $.743(255)$ & .458 & $-.38411 ; .84984$ \\
\hline & Femin. & 3.85 & .64 & & & \\
\hline \multicolumn{7}{|l|}{ COMPA-A: Filhos em relação à mãe $(\mathrm{n}=143)$} \\
\hline \multirow[t]{2}{*}{ I. Disponibilidade Parental para a Comunicação } & Masc. & 4.12 & .69 & $-1.191(256)$ & .235 & $-3.63830 ; .89663$ \\
\hline & Femin. & 4.22 & .63 & & & \\
\hline \multirow[t]{2}{*}{ II. Confiança/Partilha de Filhos para Progenitores } & Masc. & 3.65 & .78 & $-1.746(259)$ & .082 & $-2.51975 ; .15127$ \\
\hline & Femin. & 3.82 & .78 & & & \\
\hline \multirow[t]{2}{*}{ III. Expressão do Afeto e Apoio Emocional } & Masc. & 4.08 & .77 & $-1.024(262)$ & .307 & $-1.46006 ; .46070$ \\
\hline & Femin. & 4.18 & .80 & & & \\
\hline \multirow[t]{2}{*}{ IV. Metacomunicação } & Masc. & 3.78 & .72 & $-1.022(259)$ & .308 & $-2.33560 ; .73927$ \\
\hline & Femin. & 3.87 & .68 & & & \\
\hline \multirow[t]{2}{*}{ V. Padrão Comunicacional Negativo } & Masc. & 3.83 & .70 & $-1.125(262)$ & .261 & $-1.01694 ; .27727$ \\
\hline & Femin. & 3.93 & .63 & & & \\
\hline \multicolumn{7}{|l|}{ COMPA-P: Pais $(\mathrm{n}=55)$; Mães $(\mathrm{n}=271)$} \\
\hline \multirow[t]{2}{*}{ I. Expressão do Afeto e Apoio Emocional } & Masc. & 4.22 & .39 & $-2.609(317)$ & $.010^{*}$ & $-3.14443 ;-.44090$ \\
\hline & Femin. & 4.37 & .38 & & & \\
\hline \multirow[t]{2}{*}{ II. Disponibilidade Parental para a Comunicação } & Masc. & 4.07 & .49 & $.470(288)$ & .639 & $-.87388 ; 1.42253$ \\
\hline & Femin. & 4.03 & .48 & & & \\
\hline \multirow[t]{2}{*}{ III. Metacomunicação } & Masc. & 4.24 & .43 & $-.495(321)$ & .621 & $-1.35607 ; .81108$ \\
\hline & Femin. & 4.28 & .46 & & & \\
\hline \multirow[t]{2}{*}{ IV. Confiança/Partilha de Progenitores para Filhos } & Masc. & 3.86 & .51 & $-.988(323)$ & .324 & $-1.56479 ; .51832$ \\
\hline & Femin. & 3.94 & .51 & & & \\
\hline \multirow[t]{2}{*}{ V. Confiança/Partilha de Filhos para Progenitores } & Masc. & 3.78 & .51 & $-2.417(326)$ & $.016^{*}$ & $-2.13268 ;-.21857$ \\
\hline & Femin. & 3.95 & .46 & & & \\
\hline
\end{tabular}

${ }^{*} \mathrm{p}<.05$.

cala expressão do afeto e apoio emocional [ $t$ (317) $=-2.609, p=.010]$, com uma magnitude do efeito média $\left(\eta^{2}=.06\right)$, e da subescala confiança/partilha comunicacional de filhos para progenitores $[t$ (326) $=-2.417, p=.016]$, com uma magnitude do efeito média $\left(\eta^{2}=.06\right)$. Em média, as mães destacam-se por terem uma melhor perceção nas dimensões relacionadas com o apoio emocional e afetivo $(M=4.37, D P=.38)$ e confiança/partilha comunicacional por parte dos filhos $(M=$ $4.22, D P=.39)$, comparativamente aos pais $(M=$ 3.78, $D P=.51 ; M=3.95, D P=.46)$.

\section{Subobjetivo 2: Analisar se o nível de conflito com as figuras parentais é independente do sexo dos adolescentes}

A análise efetuada para responder ao segundo subobjetivo não revelou diferenças estatisticamente significativas entre os adolescentes do sexo masculino (conflito em relação ao pai: $M=$ $3.91, D P=.61$; conflito em relação à mãe: $M=$ 3.83, $D P=.70$ ) e do sexo feminino (conflito em relação ao pai: $M=3.85, D P=.64$; conflito em relação à mãe: $M=3.93, D P=.63$ ) em relação ao conflito comunicacional com ambos os progenitores (em relação ao pai $[t(255)=0.743, p$ $=.458]$, em relação à mãe $[t(262)=-1.125, p=$ .261]) (Tabela 1). 
Subobjetivo 3: Analisar a influência das variáveis escolaridade, área de residência, nível socioeconómico e estrutura familiar de progenitores e adolescentes na perceção dos padrões comunicacionais entre pais e filhos

Para analisar a influência das variáveis escolaridade, área de residência, nível socioeconómico e estrutura familiar de progenitores e adolescentes na comunicação parento-filial recorreu-se ao teste ANOVA one-way e ao teste de Tukey para comparação múltipla das médias. Estes resultados podem ser consultados nas Tabelas 2 e 3 .

\section{Resultados na amostra dos adolescentes}

Os resultados sugerem a existência de diferenças estatisticamente significativas em função do nível socioeconómico $[F(2,251)=3.565, p=$ $.030]$ e da estrutura familiar $[F(3,252)=2.912, p$
$=.035]$. Considerando o estatuto socioeconómico, os adolescentes de estatuto socioeconómico alto tendem a percepcionar menos padrões negativos de comunicação com as suas mães $(M=3.47, D P$ $=.82$ ), comparativamente aos adolescentes de estatuto socioeconómico baixo $(M=4.03, D P=$ $.74)$ e médio $(M=3.90, D P=.64)$. Relativamente à estrutura familiar, o teste de Tukey indica que os adolescentes de famílias pós-divórcio tendem a partilhar os seus problemas e a confiar mais nas suas mães $(M=3.96, D P=.84)$ do que os adolescentes de famílias monoparentais $(M=2.82, D P$ $=.47)$. Apesar de estas diferenças serem estatisticamente significativas, o cálculo da magnitude do efeito, através do eta squared, revelou um valor de .30, significando um efeito pequeno ${ }^{15}$.

\section{Resultados na amostra dos progenitores}

Obtiveram-se diferenças estatisticamente significativas em diversas dimensões, em função

Tabela 2. Anova One-way: Diferenças na Comunicação Parento-filial ao Nível das Variáveis Sociodemográficas dos Filhos.

\begin{tabular}{|c|c|c|c|c|c|c|c|c|c|c|c|}
\hline & & \multicolumn{5}{|c|}{ COMPA-A em relação ao Pai } & \multicolumn{5}{|c|}{ COMPA-A em relação à Mãe } \\
\hline & & I. & II. & III. & IV. & $\mathrm{V}$. & I. & II. & III. & IV. & $\mathrm{V}$. \\
\hline \multicolumn{12}{|l|}{ Local de Residência } \\
\hline \multirow[t]{2}{*}{ Urbano } & M & 3.82 & 2.99 & 3.56 & 3.54 & 4.00 & 4.20 & 3.79 & 4.06 & 3.82 & 4.80 \\
\hline & $\mathrm{DP}$ & .93 & 1.09 & 1.02 & .80 & .75 & .79 & .90 & .84 & .76 & .82 \\
\hline \multirow[t]{2}{*}{ Medianamente Urbano } & $\mathrm{M}$ & 4.01 & 3.14 & 3.76 & 3.64 & 3.90 & 4.12 & 3.75 & 4.08 & 3.79 & 3.86 \\
\hline & DP & .73 & .76 & .96 & .64 & .66 & .68 & .76 & .82 & .69 & .69 \\
\hline \multirow[t]{3}{*}{ Rural } & M & 3.96 & 3.04 & 3.79 & 3.58 & 3.83 & 4.23 & 3.68 & 4.18 & 3.85 & 3.85 \\
\hline & DP & .65 & .73 & .84 & .68 & .57 & .61 & .79 & .74 & .70 & .61 \\
\hline & $\mathrm{F}$ & .601 & .572 & .552 & .359 & .785 & .880 & .252 & .473 & .256 & .973 \\
\hline \multicolumn{12}{|l|}{ Nível Socioeconómico } \\
\hline \multirow[t]{2}{*}{ Baixo } & $\mathrm{M}$ & 3.89 & 2.88 & 3.61 & 3.56 & 4.00 & 4.12 & 3.71 & 3.94 & 3.73 & 4.03 \\
\hline & DP & .63 & .73 & .95 & .63 & .76 & .63 & .93 & .95 & 0.78 & .74 \\
\hline \multirow[t]{2}{*}{ Médio } & $\mathrm{M}$ & 3.98 & 3.09 & 3.77 & 3.61 & 3.87 & 4.18 & 3.74 & 4.14 & 3.83 & 3.90 \\
\hline & DP & .74 & .79 & .93 & .68 & 2.51 & .70 & .76 & .79 & .70 & .64 \\
\hline \multirow[t]{3}{*}{ Alto } & $\mathrm{M}$ & 3.89 & 3.30 & 3.86 & 3.56 & 0.63 & 4.06 & 3.54 & 3.94 & 3.77 & 3.47 \\
\hline & $\mathrm{DP}$ & .56 & .61 & .81 & .61 & .56 & .64 & .90 & .68 & .70 & .82 \\
\hline & $\mathrm{F}$ & .227 & 1.014 & .292 & .073 & .759 & .305 & .437 & .897 & .178 & $3.565^{*}$ \\
\hline \multicolumn{12}{|l|}{ Composição Familiar } \\
\hline \multirow[t]{2}{*}{ Nuclear Intacta } & $\mathrm{M}$ & 3.99 & 3.11 & 3.80 & 3.61 & 3.88 & 4.18 & 3.71 & 4.10 & 3.80 & 3.90 \\
\hline & DP & .69 & 5.75 & .88 & .64 & .61 & .64 & .76 & .77 & .68 & .61 \\
\hline \multirow[t]{2}{*}{ Pós-Divórcio } & $\mathrm{M}$ & 3.72 & 2.82 & 3.58 & 3.61 & 3.80 & 4.26 & 3.96 & 4.29 & 3.83 & 4.01 \\
\hline & DP & .95 & .96 & 1.05 & .89 & .81 & .82 & .84 & .80 & .83 & .84 \\
\hline \multirow[t]{2}{*}{ Monoparental } & $\mathrm{M}$ & 4.18 & 3.79 & 4.10 & 3.17 & 4.63 & 3.50 & 2.82 & 4.05 & 3.22 & 3.56 \\
\hline & $\mathrm{DP}$ & .56 & .71 & .71 & .24 & .18 & .39 & .47 & .91 & .41 & .88 \\
\hline \multirow[t]{3}{*}{ Reconstituída } & $\mathrm{M}$ & 4.03 & 3.01 & 3.60 & 3.63 & 3.83 & 4.08 & 3.65 & 4.10 & 3.90 & 3.62 \\
\hline & DP & .66 & .81 & 1.03 & .71 & .58 & .67 & .81 & .90 & .65 & .80 \\
\hline & $\mathrm{F}$ & 1.140 & 1.517 & .848 & .293 & 1.111 & 1.735 & $2.912^{*}$ & .550 & 1.662 & 2.078 \\
\hline
\end{tabular}

$\mathrm{p}<.05$. 
Tabela 3. Anova One-way: Diferenças na Comunicação Parento-filial ao Nível das Variáveis Sociodemográficas dos Progenitores.

\begin{tabular}{|c|c|c|c|c|c|c|}
\hline & & I. & II. & III. & IV. & V. \\
\hline \multicolumn{7}{|l|}{ Local de Residência } \\
\hline \multirow[t]{2}{*}{ Urbano } & M & 4.32 & 4.03 & 4.27 & 3.79 & 3.96 \\
\hline & DP & .32 & .42 & .35 & .45 & .41 \\
\hline \multirow[t]{2}{*}{ Medianamente Urbano } & $\mathrm{M}$ & 4.34 & 4.06 & 4.24 & 3.99 & 3.90 \\
\hline & DP & .45 & .54 & .52 & .54 & .51 \\
\hline \multirow[t]{3}{*}{ Rural } & $\mathrm{M}$ & 4.40 & 4.03 & 4.38 & 4.05 & 3.92 \\
\hline & DP & .32 & .42 & .43 & .51 & .53 \\
\hline & $\mathrm{F}$ & .601 & .133 & 1.597 & $6.515^{*}$ & .626 \\
\hline \multicolumn{7}{|l|}{ Nível Socioeconômico } \\
\hline \multirow[t]{2}{*}{ Baixo } & $\mathrm{M}$ & 4.34 & 4.05 & 4.19 & 3.99 & 3.85 \\
\hline & DP & .48 & .51 & .60 & .57 & .54 \\
\hline \multirow[t]{2}{*}{ Médio } & $\mathrm{M}$ & 4.33 & 4.02 & 4.29 & 3.89 & 3.93 \\
\hline & DP & .35 & .47 & .39 & .49 & .44 \\
\hline \multirow[t]{3}{*}{ Alto } & $\mathrm{M}$ & 4.46 & 4.29 & 4.44 & 4.02 & 4.19 \\
\hline & DP & .29 & .46 & .37 & .43 & .50 \\
\hline & $\mathrm{F}$ & .791 & 2.627 & 2.845 & 1.552 & $3.851^{*}$ \\
\hline \multicolumn{7}{|l|}{ Composição Familiar } \\
\hline \multirow{2}{*}{ Nuclear Intacta } & $\mathrm{M}$ & 4.34 & 4.05 & 4.26 & 3.91 & 3.92 \\
\hline & DP & .39 & .49 & .46 & .51 & .49 \\
\hline \multirow[t]{2}{*}{ Pós-Divórcio } & $\mathrm{M}$ & 4.42 & 4.00 & 4.38 & 4.09 & 3.99 \\
\hline & $\mathrm{DP}$ & .31 & .48 & .34 & .52 & .38 \\
\hline \multirow[t]{2}{*}{ Monoparental } & M & 4.28 & 3.94 & 4.16 & 3.86 & 3.91 \\
\hline & DP & .49 & .41 & .59 & .57 & .46 \\
\hline \multirow{3}{*}{ Reconstituída } & $\mathrm{M}$ & 4.12 & 3.78 & 4.33 & 3.86 & 3.77 \\
\hline & $\mathrm{DP}$ & .24 & .36 & .24 & .36 & .40 \\
\hline & $\mathrm{F}$ & 1.206 & .742 & 1.069 & 1.471 & .450 \\
\hline \multicolumn{7}{|l|}{ Nível de Escolaridade } \\
\hline \multirow{2}{*}{4 anos } & $\mathrm{M}$ & 4.25 & 4.00 & 4.05 & 3.93 & 3.77 \\
\hline & DP & .65 & .47 & .78 & .58 & .50 \\
\hline \multirow[t]{2}{*}{6 anos } & $\mathrm{M}$ & 4.46 & 4.24 & 4.05 & 4.04 & 3.95 \\
\hline & DP & .42 & .53 & .47 & .59 & .63 \\
\hline \multirow[t]{2}{*}{9 anos } & $\mathrm{M}$ & 4.44 & 4.18 & 4.24 & 4.14 & 3.94 \\
\hline & DP & .40 & .43 & .47 & .56 & .55 \\
\hline \multirow[t]{2}{*}{12 anos } & M & 4.36 & 4.05 & 4.23 & 3.97 & 4.02 \\
\hline & DP & .35 & .57 & .39 & .45 & .42 \\
\hline \multirow[t]{2}{*}{ Licenciatura } & M & 4.29 & 3.94 & 4.27 & 3.84 & 3.91 \\
\hline & DP & .35 & .48 & .40 & .47 & .44 \\
\hline \multirow[t]{2}{*}{ Mestrado } & $\mathrm{M}$ & 4.26 & 4.04 & 4.42 & 3.73 & 3.91 \\
\hline & DP & .26 & .35 & .24 & .36 & .33 \\
\hline \multirow[t]{3}{*}{ Doutoramento } & $\mathrm{M}$ & 4.38 & 4.06 & 4.03 & 3.86 & 4.05 \\
\hline & DP & .31 & .45 & .37 & .60 & .39 \\
\hline & $\mathrm{F}$ & 1.388 & 1.659 & $2.181^{*}$ & $2.494^{*}$ & 1.206 \\
\hline
\end{tabular}

${ }^{*} \mathrm{p}<.05$.

de algumas variáveis sociodemográficas. Considerando o nivel de escolaridade dos progenitores, registraram-se diferenças estatisticamente significativas nas subescalas metacomunicação $[F(7,308)=2.181, p=.036]$ e confiança/partilha comunicacional de progenitores para filhos $[F(7$, $309)=2.494, p=.017]$. Em concreto, estas diferenças indicam que os progenitores que comple- 
do $(M=3.73, D P=.36)$. A magnitude do efeito para estas diferenças é pequeno $\left(\eta^{2}=.02\right)$.

$\mathrm{Na}$ análise da escala COMPA em função da variável área de residência dos progenitores registaram-se diferenças estatisticamente significativas na dimensão confiança/partilha comunicacional de progenitores para filhos $[F(2,315)=6.515, p$ $=.002]$, no sentido dos progenitores que vivem num contexto predominantemente urbano percecionarem uma menor confiança/partilha nos seus filhos $(M=3.79, D P=.45)$ comparativamente aos que vivem em contextos medianamente urbanos $(M=3.99, D P=.54)$ e predominantemente rurais $(M=4.05, D P=.51)$. Apesar disto, o tamanho de este feito é pequeno $\left(\eta^{2}=.02\right)$.

O nível socioeconómico foi outra variável analisada na amostra dos progenitores, registrando diferenças estatisticamente significativas na dimensão confiança/partilha comunicacional de filhos para progenitores $[F(2,320)=3.851, p=$ $.022]$. Os resultados sugerem que os progenitores de estatuto socioeconómico alto percepcionam uma maior partilha e confiança por parte dos filhos $(M=4.19, D P=.50)$, comparativamente aos de estatuto baixo $(M=3.58, D P=.54)$. A magnitude do efeito para estes resultados é, em todos os casos, pequeno $\left(\eta^{2}=.01\right)$.

Por fim, a variável estrutura familiar não revelou qualquer diferença estatisticamente significativa, nem na escala total, nem nas subescalas.

\section{Discussão}

O objetivo da presente investigação consistiu na análise das perceções de progenitores e de adolescentes sobre a comunicação mantida na relação parento-filial, através da aplicação da escala COMPA. Pretendia-se caracterizar a comunicação entre pais e filhos adolescentes atendendo a três subobjetivos. Para tal, realizaram-se análises estatísticas paramétricas cujos resultados serão seguidamente discutidos, analisando a sua concordância ou divergência com outras pesquisas já publicadas.

Começando pelas diferenças nos padrões comunicacionais em função da variável sexo, constatou-se que os rapazes tendem a confiar e a partilhar as suas dificuldades com as figuras parentais do sexo masculino, enquanto as adolescentes fazem-no, preferencialmente, mas não de forma estatisticamente significativa, com as mães. Este resultado contradiz parcialmente a literatura que indica que as mães tendem a ser mais procuradas para conversar ${ }^{9,10}$.
No entanto, uma pesquisa mais cuidada da literatura ${ }^{6,16}$ revela a existência de estudos que corroboram os resultados da presente investigação. Grotevant e Cooper ${ }^{16}$ constataram que os pais, mais do que as mães, tendem a encorajar a assertividade dos adolescentes, quando da crise identitária. No estudo de Tomé et al. ${ }^{6}$ verificou-se que os rapazes comunicam mais com os progenitores e amigos enquanto que as adolescentes do sexo feminino comunicam melhor com o grupo de pares do que com os progenitores. Contudo, na perspetiva dos progenitores continua a verificarse uma perceção mais positiva das mães, comparativamente aos pais, em relação à comunicação que mantêm com os filhos adolescentes ao nível da disponibilidade para a comunicação, da metacomunicação e da perceção sobre a confiança/ partilha por parte dos filhos ${ }^{9}$. Este resultado pode advir das representações sociais que favorecem alguns estereótipos relativamente às figuras parentais: por um lado, o papel protetor e afetivo das mães e, por outro, o papel disciplinador e patriarca dos pais ${ }^{17}$.

Considerando o conflito entre progenitores e filhos adolescentes não se verificaram diferenças em função da variável sexo, resultado que contraria a literatura empírica revista ${ }^{10}$. Os padrões negativos de comunicação são percepcionados pelos adolescentes de ambos os sexos como sendo pouco frequentes, o que não quer dizer que não existam. Este resultado poderá explicar-se pelo facto de, atualmente, os temas/problemáticas que favorecem os conflitos parento-filiais serem cada vez mais transversais aos adolescentes de ambos os sexos (e.g., autorização parental para saídas noturnas, relação com o grupo de pares, utilização excessiva das novas tecnologias, (in) sucesso escolar, emancipação sexual, consumo de substâncias, regras familiares). Exemplo disto é o estudo realizado por Villa Moral-Jiménez et al. ${ }^{18}$ que analisou as diferenças atitudinais em função do sexo dos adolescentes no consumo de substâncias psicoativas. Esta investigação demonstrou que, apesar das adolescentes manifestarem uma atitude de maior sensibilização perante os efeitos negativos do consumo destas substâncias e os rapazes manifestarem uma atitude permissiva, ambos referem disponibilidade e curiosidade para experimentar drogas. O estudo sobre os consumos de este tipo de substâncias por parte dos adolescentes foi associado, durante algum tempo e em exclusivo, ao sexo masculino ${ }^{19}$.

Os resultados obtidos nas duas versões (parental e filial) da escala COMPA indicam que algumas variáveis sociodemográficas têm um papel 
importante na comunicação entre progenitores e filhos. Os dados mais significativos surgiram na amostra dos progenitores. De acordo com o que se previa, atendendo à literatura, quando os progenitores têm níveis de escolaridade baixos, a frequência comunicacional com os seus filhos adolescentes tende a ser menor ${ }^{10}$. Os progenitores que completaram apenas o $6^{\circ}$ ano de escolaridade tendem a metacomunicar menos com os seus filhos do que os progenitores com níveis superiores de escolaridade. Uma menor escolaridade poderá traduzir-se em competências linguísticas mais pobres nos progenitores, dificultando a estruturação de um pensamento complexo e a construção de uma comunicação clara ${ }^{20}$. Verificou-se ainda que os progenitores com maiores níveis de escolaridade tendem a percepcionar maior confiança e partilha de problemas por parte dos seus filhos do que aqueles que têm níveis de escolaridade mais baixos. Este resultado é convergente com a ideia defendida por Crouter e Head ${ }^{21}$ indicando que quanto maior for a escolaridade dos progenitores, mais informados estes estarão sobre as experiências quotidianas dos seus filhos. Ainda assim, os resultados revelaram um dado curioso: progenitores com níveis de escolaridade mais baixos tendem a estar mais disponíveis para comunicar com os seus filhos adolescentes do que os licenciados. Esses progenitores manifestam, igualmente, uma maior confiança nos filhos e referem partilhar intimidades/problemas com eles, em comparação com os que têm licenciatura, mestrado e doutoramento. Uma possível explicação para este resultado pode ser a existência de uma maior liberdade e espontaneidade comunicacional nos progenitores com níveis escolares baixos/médios. Considerando as questões culturais, supõe-se que a existência de qualificações superiores limita a expressão de uma comunicação mais genuína, aberta e livre de preconceitos.

Apesar da escassez de investigação sobre a influência do estatuto socioeconómico na comunicação entre pais e filhos adolescentes, alguns autores $^{22}$ indicam que, à semelhança do que acontece com o nível de escolaridade, quanto mais elevado é o estatuto socioeconómico do progenitor, melhor tende a ser a sua comunicação com o adolescente, marcada por um estilo parental democrático. Os resultados do presente estudo são congruentes com esta hipótese, verificandose que os progenitores de estatuto socioeconómico alto percecionam maior confiança e partilha comunicacional por parte dos filhos comparativamente aos progenitores de estatuto socioeconómico baixo e médio. Estes resultados são com- preensíveis considerando que, por norma, os indivíduos de estatuto socioeconómico baixo têm menor escolarização e, por isso, menor capacidade de elaboração ao nível comunicacional ${ }^{23}$. Os resultados obtidos revelam a fragilidade interacional nas famílias de estatutos socioeconómicos desfavorecidos, tal como demonstra o estudo de Conger e Conger ${ }^{22}$. Estes autores sugerem que as dificuldades económicas desencadeiam respostas emocionais de stress que afetam a qualidade do exercício da parentalidade que, por sua vez, pode facilitar o desajustamento social dos filhos adolescentes. Os resultados da presente investigação corroboram os dados obtidos pelo estudo de Zhang ${ }^{23}$, ou seja, filhos de famílias desfavorecidas tendem a estabelecer uma relação mais conflituosa com os seus progenitores do que filhos de famílias de estatuto socioeconómico médio ou alto. $\mathrm{O}$ autor considera que estes resultados se explicam atendendo ao impacto adverso que a pobreza tem sobre as emoções e os comportamentos dos progenitores. O estudo de Conger e Conger $^{22}$ revela, também, que os progenitores de nível socioeconómico alto tendem a percepcionar maior confiança e partilha de intimidades por parte dos seus filhos do que os progenitores de estatuto socioeconómico baixo ou médio. Os mesmos autores enquadram este resultado indicando que a monitorização parental tende a ser mais rígida e punitiva nas famílias com baixos níveis de rendimento do que nas de níveis médios ou altos, criando condições favoráveis a que os adolescentes se sintam menos confortáveis para partilhar tópicos problemáticos e confiar aspetos da sua intimidade com os progenitores.

Considerando a variável área de residência, os dados indicam que os progenitores que habitam num contexto predominante urbano tendem a confiar e a partilhar menos os seus problemas/ preocupações/intimidades com os filhos adolescentes, comparativamente aos que vivem em contextos medianamente urbanos e predominantemente rurais. Apesar da literatura existente sobre este tema ser escassa e dispersa ${ }^{24}$, este é um resultado esperado. Considerando as características da sociedade urbana moderna (longa jornada de trabalho, distância entre o trabalho e a casa, pouco tempo de convívio com os filhos), depreende-se que estes progenitores tenham menos disponibilidade para estar física e afetivamente com os filhos limitando a oportunidade de partilha de assuntos do foro pessoal. $\mathrm{O}$ isolamento e a privação de alguns serviços nos contextos rurais podem conduzir à rigidificação dos modelos educativos e comunicacionais entre pais e ado- 
lescentes ${ }^{24}$, porém, numa vertente mais positiva, pode facilitar a partilha genuína das preocupações parentais.

Por fim, um dos resultados mais surpreendentes obtidos com a escala COMPA relaciona-se com a variável estrutura familiar. Ao contrário de algumas indicações da literatura ${ }^{11,25}$, não se verificaram diferenças estatisticamente significativas na subamostra dos progenitores. Por outro lado, constatou-se que os adolescentes que integram famílias pós-divórcio tendem a confiar/partilhar mais os seus problemas com as mães do que os adolescentes de famílias monoparentais. A literatura indica que, apesar de já não ser tratado como um acontecimento excecional, a separação/divórcio marca um dos períodos mais desafiantes, pois trata-se "do maior rompimento no processo de ciclo de vida familiar, aumentando a complexidade das tarefas desenvolvimentais que a família está a experienciar naquela fase" ${ }^{26}$. O estudo de Carlsund et al. ${ }^{11}$ revela que adolescentes que pertencem a agregados familiares com um único progenitor tendem a percecionar uma pior comunicação parento-filial comparativamente a adolescentes de famílias nucleares intactas. Porém, o mesmo estudo indica que a comunicação entre progenitores e filhos não difere substancialmente quando se comparam famílias nucleares intactas com famílias pós-divórcio em regime de custódia partilhada. Os autores verificaram que a partilha igualitária de responsabilidades parentais, independentemente do tipo de estrutura familiar, não parece influenciar o estabelecimento de uma comunicação parento-filial adequada. A meta-análise conduzida por Amato ${ }^{25}$, com base em 67 estudos sobre divórcio, publicados ao longo da década de 90, indica que os filhos de famílias em processo de divórcio apresentam valores significativamente mais baixos, em comparação com famílias nucleares intactas, ao nível do sucesso escolar, comportamento, ajustamento psicológico, autoconceito e manutenção de relações sociais. Apesar de estes resultados, o autor concluiu que, na década de 90, houve um decréscimo nas diferenças entre famílias nucleares intactas e famílias pós-divórcio em algumas de estas dimensões. Esta pesquisa mostra que o divórcio tem vindo a ser encarado de forma diferente pela sociedade e isso poderá explicar o resultado registado no trabalho sob discussão. Com o aumento da taxa de divórcios, a adaptação social a este acontecimento tende a ser cada vez mais normativa, sobretudo quando não se verifica conflito conjugal acentuado ${ }^{27}$. Assim, os resultados obtidos podem ser explicados pela possibilidade de os pais divorciados que integraram esta amostra terem um baixo nível de conflito interparental.

A análise dos resultados obtidos deve ser feita de modo cauteloso, uma vez que esta pesquisa apresenta algumas limitações. Salienta-se a heterogeneidade ao nível das características sociodemográficas (sexo, área de residência, nível socioeconómico, escolaridade e estrutura familiar). Esta limitação é particularmente relevante quando se comparam os resultados das famílias pós-divórcio com os outros tipos de composição familiar, dado o número reduzido de participantes. É importante ponderar que os resultados obtidos nestas variáveis podem dever-se a uma série de fatores/variáveis não controlados nesta pesquisa. Importa, também, realçar que neste estudo não foi contemplada a variável crise económica. Dada a situação financeira de Portugal e, inclusivamente, de outros países da União Europeia (e.g., Espanha, Grécia, Irlanda), seria vantajoso analisar a existência de uma eventual relação entre os dados obtidos na presente pesquisa e o panorama económico de fundo. Como foi referido anteriormente, o estatuto socioeconómico parece ter repercussões relevantes na qualidade da comunicação parento-filial ${ }^{22}$, de modo que a análise desta variável poderia permitir a clarificação dos resultados apresentados neste trabalho.

Em conclusão, do conjunto de resultados, realça-se o papel da figura paterna na perceção dos adolescentes relativamente à partilha comunicacional e, por outro lado, uma perceção mais positiva por parte das mães relativamente à expressão afetiva dos filhos e à confiança/partilha de estes. Os dados demonstram a incongruência, que por vezes existe, entre as perceções de progenitores e de adolescentes.

Apesar de circunscritos à população portuguesa, os resultados desta pesquisa permitem caracterizar a comunicação entre pais e filhos adolescentes, contribuindo assim, para o desenho de estratégias de intervenção específicas para famílias com filhos adolescentes.

Considera-se fundamental que futuramente se desenvolvam pesquisas com populações específicas/clínicas, não só para promover estudos de validade da escala COMPA, como também para compreender as particularidades da comunicação entre pais e filhos adolescentes em situações específicas (e.g., famílias em processo de regulação das responsabilidades parentais, famílias com um elemento com doença crónica). A investigação futura deve contemplar também famílias com crianças ou com jovens adultos, permitindo comparações entre as várias etapas do ciclo vital. 


\section{Colaboradores}

APM Portugal contribuiu na implementação da investigação e redação do manuscrito; e IMM Alberto na implementação da investigação e na orientação/supervisão da redação do manuscrito.

\section{Agradecimentos}

À Fundação para a Ciência e a Tecnologia e ao Ministério da Educação de Portugal pelo financiamento deste trabalho.

\section{Referências}

1. Pereira R. Introducción. In: Pereira R, organizador. Adolescentes en el siglo XXI: entre impotencia, resiliencia y poder. Madrid: Ediciones Morata; 2011. p. 11-20.

2. Carr A. Family therapy. Concepts, process and practice. $2^{\text {nd }}$ ed. Chichester: John Wiley \& Sons; 2006.

3. Preto NG. As mudanças no ciclo de vida familiar. In: Carter B, McGoldrick M. Uma estrutura para a terapia familiar. Transformações do sistema familiar na adolescência. 2a ed. Porto Alegre: Artes Médicas; 1995. p. 223-247.

4. Steinberg L, Morris A. Adolescent Development. Annu Rev Psychol 2001; 52(1):83-110.

5. Segrin C, Flora J. Family Communication. London: Lawrence Erlbaum Associates; 2005.

6. Tomé G, Matos M, Camacho I, Simões C, Diniz J. Portuguese adolescents: The importance of parents and peer groups in positive health. Span J Psychol 2012; 15(3):1315-1324.

7. Herbert M. Handbook of parenting. Theory and research for practice. Parenting across the lifespan. London: Sage Publications; 2004.

8. Laursen B, Collins WA. Handbook of family communication, Parent-child communication during adolescence. New Jersey: Lawrence Erlbaum Associates; 2004. p. 333-348.

9. Barnes H, Olson D. Parent-Adolescent Communication and the Circumplex Model. Child Dev 1985; 56(2):438-447.

10. Jiménez AP, Delgado AO. Comunicación y conflicto familiar durante la adolescencia. An Psicol 2002; 18(2):215-231.

11. Carlsund A, Eriksson U, Sellström E. Shared physical custody after family split-up: implications for health and well-being in Swedish schoolchildren. Acta Paedia$\operatorname{tr} 2013$; 102(3):318-323.

12. Cloutier R, Filion L, Timmermans H. Quando os pais se separam... Para melhor lidar com a crise e ajudar a criança. Lisboa: Climepsi Editores; 2006.

13. Alarcão M. (Des)Equilíbrios Familiares. $3^{\text {a }}$ ed. Coimbra: Quarteto; 2006

14. Portugal A, Alberto I. Escala de Avaliação da Comunicação na Parentalidade (COMPA): Desenvolvimento e validação de uma medida da comunicação parento-filial. Av Piscol Latinonot 2014; 32(1):85-103.

15. Pallant J. SPSS Survival Manual. A step by step guide to data analysis using SPSS for program (Version 18). $4^{\text {th }}$ ed. Philadelphia: Open University Press; 2010.

16. Grotevant $\mathrm{H}$, Cooper C. Patterns of Interaction in Family Relationships and the Development of Identity Exploration in Adolescence. Child Dev 1985; 56(2): 415-428.

17. Park RD. Handbook of Parenting. Being and becoming a parent. Fathers and families. $2^{\text {nd }}$ ed. London: Lawrence Erlbaum Associates; 2002. Vol 3.

18. Villa Moral-Jiménez M, Ovejero-Bernal A, Castro Á, Rodríguez-Díaz F, Sirvent-Ruiz C. Modificación de actitudes hacia el consumo de sustanciasen adolescentes: Seguimiento de las diferencias inter-género. Int J Clin Helath Psyc 2011; 11(2):291-311.

19. Biederman J. Familial risk analysis of the association between attention-deficit/hyperactivity disorder and psychoactive substance use disorder in female adolescents: a controlled study. J Child Psychol Psyc 2009; 50(3):352-358. 
20. Hoff E, Laursen B, Tardif T. Handbook of parenting. Biology and ecology of parenting.Socioeconomic status and parenting. $2^{\text {nd }}$ ed. London: Lawrence Erlbaum Associates; 2002. Vol. 2. p. 231-252.

21. Crouter AC, Head MR. Handbook of parenting. Being and becoming a parent. Parental monitoring and knowledge of children. $2^{\text {nd }}$ ed. London: Lawrence Erlbaum Associates; 2002. Vol. 3. p. 461-483.

22. Conger R, Conger K. A family process model of economic hardship and adjustment of early adolescent boys. Child Dev 1992; 63(3):526-541.

23. Zhang X. The Effects of Parental Education and Family Income on Mother-Child Relationships, Father-Child Relationships, and Family Environments in the People's Republic of China. Fam Process 2012; 51(4):483497.

24. Diggs RC, Socha T. Communication, families, and exploring the boundaries of cultural diversity. In: Vangelisti AL, editor. Handbook of family communication. New Jersey: Lawrence Erlbaum Associates; 2004. p. 249-266.

25. Amato P. Children of divorce in the 1990s: An update of the Amato and Keith (1991) meta-analysis. J Fam Psychol 2001; 15(3):355-370.

26. Peck JS, Manocherian JR. As Mudanças no Ciclo de Vida Familiar. Uma estrutura para a terapia familiar. $4^{\mathrm{a}}$ ed. In: Peck JS, Manocherian JR. O Divórcio nas mudanças do ciclo de vida familiar. Porto Alegre: Artes Médicas; 1995. p. 291-320.

27. Esmaeili N, Yaacob S, Juhari R, Mariani M. Post-divorce parental conflict, economic hardship and academic achievement among adolescents of divorced families. Asia Soc Sci 2011; 7(12):119-124.

Artigo apresentado em 17/04/2014

Aprovado em 21/09/2014

Versão final apresentada em 23/09/2014 